\title{
Incertidumbre durante la atención enfermera en el cuidado del orificio de salida sano del catéter peritoneal
}

\author{
Miguel Núñez Moral \\ Hospital Universitario Central de Asturias. Oviedo. España
}

Como citar este artículo:

Núñez-Moral M. Incertidumbre durante la atención enfermera en el cuidado del orificio de salida sano del catéter peritoneal.

Enferm Nefrol. 2021 Abr-Jun;24(2):195-97

Estimado editor jefe de la revista Enfermería Nefrológica,

Desde mi responsabilidad como vocal de diálisis peritoneal de la Sociedad Española de Enfermería Nefrológica (SEDEN), me gustaría aportar unas reflexiones acerca de la cura del orificio de salida sano del catéter peritoneal para centrar el enfoque de la evidencia aplicada a la práctica clínica. Uno de los objetivos de la atención enfermera en el ámbito de la diálisis peritoneal es prevenir y tratar las infecciones relacionadas con el catéter, para evitar una complicación mayor, la peritonitis. Como postula la International Society Peritoneal Dialysis (ISPD) en $2017^{1}$.

Objetivo indiscutible porque la infección del orificio de salida (IOS) "per se" no entraña gravedad, salvo porque puede conducir a la tunelitis y/o infección peritoneal (IP) dependiente de catéter, que suele acarrear retirada de este, salida de la técnica dialítica (temporal o definitiva) y un daño de la membrana peritoneal. Lo que es más discutible es como conseguirlo debido a la propia incertidumbre de la evidencia científica en cuanto a qué hacer y cómo. Tras la inserción del catéter muchos autores ${ }^{2}$ recomiendan curas semanales porque el aumento injustificado de curas del orifico sano (OS) solo lo expone a infecciones, teniendo en cuenta que tampoco es necesario su evaluación diaria. El pronós-

\section{Correspondencia:}

Miguel Núñez Moral

E-mail: nmoral76@hotmail.com tico de una IOS no varía si se diagnostica con 24 o 48 horas de diferencia. En este sentido, la incertidumbre en cuanto a la cura del OS no aporta mas claridad, la ISPD recomienda su cuidado de mantenimiento dos veces por semana y siempre después de la ducha. Además, recomienda el uso diario de antibiótico tópico para prevenir la IOS, provocando una situación difícil de aplicar debido a la antítesis de ambas recomendaciones y la falta de evidencias solidas que apoyen una práctica de calidad y segura para el paciente. En casos de infección, se aconseja la cura diaria exclusivamente para la limpieza del exudado y valoración visual de la evolución sin que exista evidencia de que esta intervención pueda garantizar una mejora de los resultados clínicos.

La ISPD aporta luz recomendando la cura diaria con antibiótico (Ab), especialmente con mupirocina, porque reduce en un $60-70 \%$ las IOS producidas por bacterias gram+ ( $S$. aureus) y su uso diario parece que provoca menos resistencias que el uso alterno (Tabla 1). Sin embargo, estos hallazgos no permiten deducir que el uso intermitente de antibióticos produzca más o menos resistencias que la diaria. Además, debemos tener en cuenta que esta evidencia, en general, es obsoleta y presenta serias limitaciones metodológicas, siendo inexistente la comparación entre la cura diaria o alterna con antibiótico y cómo repercute en la aparición de resistencias y/o efectos no deseados.

La revisión sistemática de Obata Y. et al de $2020^{3}$ explica que la ISPD basó las recomendaciones del uso diario de mupirocina en resultados que pueden dar lugar a una interpretación engañosa sobre el establecimiento de las directrices. Sus conclusiones exponen que no hubo evidencia suficiente para recomendar la aplicación ru- 
Tabla 1. Resumen de los artículos referenciados para uso diario de mupirocina.

\begin{tabular}{|c|c|c|c|}
\hline AUTORES & AÑo & METODOLOGÍA & CONCLUSIONES \\
\hline 1 Aykut S, et al & 2010 & $\begin{array}{l}\text { n } 33 \text { p } \\
\text { seguimiento } 3 \text { años } \\
\text { Grupo } 1 \text { mup } 1 \text { vez/s } \\
\text { Grupo } 2 \text { mup } 3 \text { veces/s }\end{array}$ & $\begin{array}{l}\text { Grupo 1: } 13 \text { IP } 7 \text { IOS } \\
\text { Resis 2,11\% } \\
\text { Grupo 2: } 6 \text { IP } 1 \text { IOS } \\
\text { Resis 0,2\% }\end{array}$ \\
\hline 2 Lobbedeez T, et al & 2004 & $\begin{array}{l}\text { n } 147 \text { p } \\
\text { mup uso diaria o } 3 \text { veces/s } \\
7 \text { años comparativa }\end{array}$ & $\begin{array}{l}\text { Resis de } 2,7 \% \text { pero no diferencia } \\
\text { entre aplicación diaria o intermitente }\end{array}$ \\
\hline 3 Pérez-Fontán $M$, et al & 2002 & n 155 p seguimiento $1990-2000$ & $\begin{array}{l}\text { Evalúa resistencias tras tratamiento } \\
\text { erradicador en portadores nasales }\end{array}$ \\
\hline 4 Annigeri $R$, et al & 2001 & n 149 p uso profiláctico de mupirocina & $\begin{array}{l}\text { Resis alto nivel } 3 \% \\
1 \text { caso resis en el } 0 \text { S }\end{array}$ \\
\hline 5 Al-Hwiesh AK, et al & 2013 & $\begin{array}{l}\text { n } 203 \text { p. } 2 \text { centros } \\
1 \text { cura diaria mup } \\
1 \text { cura diaria gent }\end{array}$ & $\begin{array}{l}\text { Resis mup } 16 \% \\
\text { Menos IOS en grupo gent }\end{array}$ \\
\hline
\end{tabular}

n: numero; p: pacientes; mup: mupirocina; vez/s: aplicación veces/semana; IP: infección peritoneal; IOS: infección del orificio de salida; Resis: resistencia; 0S: orificio de salida; gent: gentamicina.

El artículo 1 compara la cura 1 vez a la semana y 3 veces a la semana, concluyendo que a más frecuencia, menos infecciones. El porcentaje de resistencias es inferior al de los trabajos 4 y 5 (cura diaria), ya que el 2 no diferencia entre el grupo de curas de 3 veces por semana y el diario. El 5 (trabajo más reciente) evalúa la utilización diaria de mupirocina en comparación con gentamicina (en 2 centros), notificando $16 \%$ de aparición de resistencias y mejor acción profiláctica de la gentamicina.

tinaria de antibióticos tópicos en el sitio de salida para la prevención de infecciones relacionadas con la diálisis peritoneal. Además, ningún estudio ha evaluado los efectos secundarios a largo plazo de la aplicación de antibióticos en el sitio de salida.

Según el Plan de calidad científico-técnico y de mejora continua de calidad en diálisis peritoneal ${ }^{4}$ las tasas de IOS varían entre 0,05 y 1,02 episodio/paciente-año. Aconsejan que la ratio de la unidad sea menor a un episodio cada 24 pacientes/mes. Parece lógico pensar que en aquellas unidades con una incidencia de IOS elevada y que la flora responsable de estas IOS sean gram+, especialmente S. aureus, deban utilizar la mupirocina de manera profiláctica en el $0 \mathrm{~S}$. Estas unidades deben evaluar la aparición de gérmenes resistentes y la variación de la flora habitual responsable de las IOS. Según la teoría del nicho ecológico (George E. Hutchinson) si modificamos un hábitat se desplaza un germen, pero puede favorecer la aparición de otros: hongos, Coryenebacteriúm, etc.

Tal y como comencé diciendo al principio, la propia naturaleza de la evidencia no aporta certidumbre en estos momentos para mejorar la toma de decisión en el cuidado del catéter, ya sea sano o infectado. Además, la vigilancia continua en la aparición de resistencias, alergias, dermatitis y cambios no deseados en la flora responsable de las IOS resulta crucial para el devenir de los resultados clínicos del paciente en diálisis peritoneal.

La frecuencia de la cura del OS, con la evidencia conocida, creo que debería limitarse a 2 veces por semana y/o después de la ducha, porque:

- La cura diaria penaliza con más "trabajo" al paciente, sin evidencia de beneficio.

- Los productos para la limpieza del OS son muy variados: hipoclorito sódico, ácido acético, suero salino hipertónico, miel etc.

Utilizarlos de manera diaria altera el $\mathrm{Ph}$ de la piel, pudiendo provocar dermatitis y alergias en la zona peri-catéter.

No nos debemos olvidar que levantar diariamente apósitos adhesivos es irritativo para la piel. Además, la mupirocina daña los catéteres de poliuretano y puede provocar eczemas, como otras pomadas tópicas (Ej Nitrofural, Furacin ${ }^{\circledR}$ )

- La cura diaria dificulta la cicatrización de un OS con pocas semanas, primero por el propio "arrastre de la cura" y por la variación de la temperatura alrededor de la herida. Esta debe ser de $37^{\circ} \mathrm{C}$, pero si disminuye provoca una vasoconstricción, dificultando 
el aporte de leucocitos a la herida y una alteración en el transporte de oxígeno y nutrientes. El contacto de la herida con el ambiente hace que disminuya su temperatura, tardando varias horas en recuperar su actividad reparadora y cicatricial ${ }^{5,6}$.

Recepción: 14-06-21

Aceptación: 20-06-21

Publicación: 30-06-21

\section{Bibliografía}

1. Szeto CC, Li PK, Johnson DW, Bernardini J, Dong $J$, Figuereido AE et al. ISPD catheter-related infection recomendations: 2017 update. Perit Dial Int. 2017;37(2):141-54.

2. Minguela JI, Lanuza M, Martínez A. Complicaciones del túnel y orificio de salida del catéter peritoneal. En Montenegro-Martinez J, Correa-Rotter R, Carlos RieIla M. Tratado de diálisis peritoneal. Editoral Elsevier (3ª edición). 2020. p. 278-9.

3. Obata $Y$, Murashima M, Toda N, Yamamoto $S$, Tsujimoto $Y$, Tsujimoto $Y$ et al. Topical application of mupirocin to exit sites in patients on peritoneal dialysis: a systematic review and meta-analysis of randomized controlled trials. Ren Reemplazar Ther. 2020 [consultado 12 jun 2021] 6,12. Disponible en: https://doi.org/10.1186/s41100-020-00261-4.

4. Bajo MA, Selgas R, Remón C, Arrieta J, Álvarez-Ude $F$, Arenas MD et al. "Plan de calidad científico-técnica y de mejora continua de calidad en diálisis peritoneal" Nefrologia. 2010;30(1):28-45.

5. Vallejo L. Siete errores comunes en el diagnóstico, manejo y tratamiento de heridas crónicas [Seven common errors in the diagnosis, management and treatment of chronic wounds]. J Wound Care. 2020 Jan 1;29(LatAm sup 1):S32-6. Spanish. PMID: 31855523.

6. García-Fernández FP, Soldevilla-Ágreda JJ, Pancorbo- Hidalgo PL, Verdú-Soriano J, López-Casanova $\mathrm{P}$, Rodríguez Palma M et al. Manejo local de úlceras y heridas. Serie de documentos técnicos GNEAUPP $n^{\circ}$ III. Grupo Nacional para el Estudio y Asesoramiento de las Úlceras por Presión y Heridas Crónicas. Logroño. 2018.

Este artículo se distribuye bajo una Licencia Creative Commons Atribución-NoComercial 4.0 Internacional. https://creativecommons.org/licenses/by-nc/4.0/ 